\title{
Banana Xanthomonas Wilt Infection: The Role of Debudding and Roguing as Control Options within a Mixed Cultivar Plantation
}

\author{
Juliet Nakakawa, ${ }^{1}$ Joseph Y. T. Mugisha, ${ }^{1}$ Michael W. Shaw, ${ }^{2}$ \\ William Tinzaara, ${ }^{3}$ and Eldad Karamura ${ }^{3}$ \\ ${ }^{1}$ Department of Mathematics, Makerere University, P.O. Box 7062, Kampala, Uganda \\ ${ }^{2}$ School of Agriculture, Policy and Development, University of Reading, Agriculture Building, Whiteknights, Reading RG6 6AR, UK \\ ${ }^{3}$ Bioversity International, Plot 106, Katalima Road, P.O. Box 24384, Naguru, Kampala, Uganda
}

Correspondence should be addressed to Juliet Nakakawa; nakakawa@cns.mak.ac.ug

Received 14 September 2017; Accepted 16 November 2017; Published 13 December 2017

Academic Editor: Irena Lasiecka

Copyright (C) 2017 Juliet Nakakawa et al. This is an open access article distributed under the Creative Commons Attribution License, which permits unrestricted use, distribution, and reproduction in any medium, provided the original work is properly cited.

An optimal control framework is designed in which the use of clean planting materials, debudding, disinfection of tools, and roguing are considered as control measures of Banana Xanthomonas Wilt (BXW) within a plantation of multiple cultivars. A model for a special case of two cultivars (AAA- and ABB-genome cultivars) was analyzed. By Pontryagin's Maximum Principle, we characterized and discussed possible control strategies that substantially reduce the infection levels of BXW within a plantation of ABB- and AAA-genome cultivars. A combination of both prevention and containment controls yielded the greatest decline in the infection levels in both cultivars. Additionally, for effective BXW management, it is important to assess the endemic level of the plantation before application of controls, and once implemented, this should be maintained even when the disease is undetectable to eliminate possible resurgence.

\section{Introduction}

Banana is a major crop grown in the East and Central African region. It provides food security and income to over 20 million people in the region [1]. Since 2001, Banana Xanthomonas Wilt (BXW) has been reported as a major threat to the banana production and livelihoods of millions of people have been affected [2]. The pathogen caused by Xanthomonas campestris pv. musacearum (Xcm) was first reported in Ethiopia on Ensete, a related crop to banana in 1964 [3]. The disease is transmitted by insect vectors, birds, bats, contaminated farming tools, and infected suckers used for setting up new plantings. It attacks all the commonly grown cultivars although some are more susceptible than others. Disease symptoms include yellowing and wilting of leaves, male bud wilting, premature ripening and rotting of fruit, yellow ooze observed on the cross section cut of the pseudostem, and eventually death of the entire plant.
Since the reporting of BXW disease, scientists have disseminated information on identification based on symptoms, mode of spread, and how to implement cultural control practices that have been used to manage similar diseases such as Moko and Bugtok [4, 5]. Cultural control practices for management of BXW have been categorized as preventative or containment. Among the preventative practices, we have debudding which is the removal of the male bud by twisting with a forked stick as soon as the last cluster is formed [6]. This approach avoids contact between the tool and the potentially infected tissues. This is done within 3 weeks since flowering to prevent insect vector from transmitting the disease.

Use of clean planting materials is another preventative approach being advocated for although there are no proper screening facilities for the seed system. Farmers basically obtain suckers for new plantings either from their plantations or from neighboring plantations. Such suckers could 
be latently infected but since the available detecting tool (lateral flow device) is not yet accessible to the farmers, there is a risk of transferring latently infected plants to new plantations. Nevertheless, to reduce this risk, farmers are constantly cautioned on the use of these suckers and the need to monitor them as they develop. The third preventative approach is the disinfection of tools between plants using sodium hypochlorite solution (usually known by the trade name of the commonest brand in Uganda: Jik) or flames of fire $[5,7]$.

Two approaches have been implemented as containment practices. These are single disease stem removal (SDSR) dealing with removal of the infected stem from the mat at stool level and roguing involving removal of the entire mat from which the infected stem arose. The latter is more effective as it eliminates the risk of vertical transmission from mother stem to attached suckers. However, it is expensive to implement as it requires more labor [7]. Kubiriba et al. [8] evaluated the SDSR approach and noted that it was effective especially if the manifesting symptoms are via the inflorescence part of the plant.

Jogo et al. [9] studied the extent to which cultural control practices have been adopted by farmers and assessed the socioeconomic factors that influence adoption of these practices among smallholder farmers in Uganda. Their results indicate that among the key determinants were labor and perceived effectiveness of the technologies being implemented. This indicates knowledge gaps in identifying the combination of control options which is cheap enough to implement but causes a large decline in number of infected stems.

The best combination of cultural control practices also depends on the main production systems within the region. The two main groups of cultivars include the East African highland banana (EAHB) AAA-genome grown in western Uganda (1700 masl) and the "kayinja" (beer banana) ABBgenome cultivar grown in the central Uganda (1300 masl) [9]. The intensity of management among these cultivars is different with the former being managed intensively than the later [9]. Also ABB-genome cultivars are more susceptible to insect infection due to the greater production of sweet nectar that attracts the insects as compared to AAA-genome cultivars. These features influence BXW management and control adoption by farmers in these respective regions.

Even though application of all control approaches has been advocated, few farmers are in position to implement them. Therefore, it is necessary to examine the approaches and identify the most cost-effective combination for management of BXW disease.

The use of optimal control approaches/models allows the determination of the most cost-effective intervention. Optimal control modeling has been extensively used to understand biomedical problems and suggest optimal control combinations that will minimize the costs [10-14]. Our model focuses on determining the optimal control combination within smallholder plantations with mixed cultivars given that in banana cropping system different cultivars are managed differently and the risk of infection is also different.

Thus, we propose a Healthy-Infected model for the spread of BXW via inflorescence infection, vertical transmission, and tool-mediated spread. We formulate an optimal control model with debudding, use of clean planting material (reducing the proportion of infected suckers), roguing/SSDR, and disinfection of tools used for cultivating between and within cultivars as our control options. The optimal control model is analyzed using the Pontryagin's Maximum Principle [15-17] and numerical simulations assessing different combinations of control options. The paper is organized as follows: in Section 2, the formulation of the optimal control model for a plantation with multiple cultivars $(m)$ is presented. Mathematical analysis and numerical simulations of the optimal control model considering a special case of two cultivars are given in Section 3 and lastly conclusions drawn are given in Section 4.

\section{Model Formulation}

Consider a population of banana stems with $m$ different cultivars each subdivided into healthy $H_{i}$ and infected $I_{i}$ classes for $i=1,2,3, \ldots, m$. We assume negligible latent and incubation period for simplicity of the model, also because omission of this compartment does not entirely affect the dynamics of the disease. It is assumed that in a unified plantation several blocks of each cultivar are maintained. The effort going into maintenance of each block will depend on preference of farmers in a specific region. The healthy compartment contains all banana stems that are diseasefree but at a risk of being infected either by contaminated tools or by insect vectors during inflorescence formation. The populations per cultivar are maintained at equilibrium by a logistic function at a constant rate $r$ and a constant carrying capacity $K$. The equilibrium $r$ is a result of various activities within the plantation including replanting, desuckering, sucker emergence, and harvesting. The infected class is increased by the following scenarios.

(1) Let $\theta_{i}$ denote the proportion of healthy suckers from infected mats; then a proportion $\left(1-\theta_{i}\right)$ indicates the suckers recruited into the infected compartment arising from vertical transmission.

(2) Disease transmission by contaminated tools is modeled using mass action incidence term with $\beta_{t}$ as the coefficient of effective contact between healthy and infected stems by tools. Assuming random use of tools, spread is independent of plantation density but rather dependent on the proportion of infected stems within the plantation. Therefore, the incidence term due to contaminated tools is given as $\beta_{t} H_{i} I_{j}$ for the interaction between cultivars $i$ and $j$.

(3) Transmission by vectors via the inflorescence infection is considered to be frequency dependent, that is, $\left(\beta_{v} H_{i}, I_{j}\right) / N$ with $\beta_{v}$ as the coefficient of effective contact by vectors between cultivars $i$ and $j$. This incidence term is reasonable because spread by vectors depends on the number of flowering stems and vector activity resulting in infection at a particular time.

Once infected, infected stems showing symptoms are removed at a constant rate $\delta_{i}$ for cultivar $i$. This rate also 
depends on the available resources and farmers' desire to control that particular cultivar. In some regions within the central Uganda, farmers tend to concentrate on the cooking banana ignoring other cultivars like kayinja which tends to increase the risk of vector spread.

We now incorporate the main cultural control practices that are being implemented in the management of BXW disease into the model formulation.

(I) $u_{1}(t)$ as the control is associated with ensuring that only clean planting materials are used for replanting after mat removal or infilling of space. This involves the ability to detect and remove infected suckers from mats such that they are not used for replanting. Since there is no proper screening for the seed system, farmers rely on suckers from their plantations or neighboring plantations for replanting. It is necessary to determine whether a sucker/planting material is clean. The lateral flow device developed could be used to detect even latently infected material but currently it is not available for farmer and even then it would be expensive. $\left(1-u_{1}(t)\right)$ shows the failure to detect and remove infected suckers so that they are reluctantly used for replanting.

(II) $u_{i j}(t)$ is the control associated with disinfection of tools used during cultivation, pruning, or harvesting between cultivars $i$ and $j . u_{i i}$ shows the effort placed in controlling within cultivar spread. This involves use of sodium hypochlorite solution or heating briefly in a fire to destroy bacteria before cutting into another stem. Besides the expense of sodium hypochlorite solution, use of fire flames may not be feasible as it requires moving to the fire and is likely to damage the cutting edge by softening or embrittling the blade depending on how often it is used. $\left(1-u_{i j}(t)\right)$ indicates failure to disinfect tools when cutting into cultivars $i$ and $j$.

(III) $u_{2}(t)$ is the control associated with debudding (removal of male bud by forked stick). This requires continuous surveillance and monitoring such that the male buds are removed immediately after the last cluster is formed to eliminate vector spread. $\left(1-u_{2}(t)\right)$ represent failure to implement this control which results in vector spread.

(IV) Roguing or single stem removal of infected stem. Let $u_{3}(t)$ indicate control associated with removal of infected stems with $\delta_{i}$ as the roguing rate of cultivar $i$. This involves detecting BXW infected stems and removing them effectively without further spread by tools or mat removal.

Combining all the cases above, the following system can be used to describe the dynamics of BXW when control measures are being implemented.

$$
\begin{aligned}
\frac{d H_{i}}{d t}= & r\left(1-\frac{N_{i}}{K}\right)\left(H_{i}+\left(1-u_{1}\right) \theta_{i} I_{i}\right) \\
& -\frac{\left(1-u_{2}\right) \beta_{v} H_{i}}{N} \sum_{j=1}^{m} I_{j}-\beta_{t} H_{i} \sum_{j=1}^{m}\left(1-u_{i j}\right) I_{j},
\end{aligned}
$$

$$
\begin{aligned}
\frac{d I_{i}}{d t}= & r\left(1-\frac{N_{i}}{K}\right)\left(1-\left(1-u_{1}\right) \theta_{i}\right) I_{i} \\
& +\frac{\left(1-u_{2}\right) \beta_{v} H_{i}}{N} \sum_{j=1}^{m} I_{j}-\delta_{i} u_{3} I_{i} \\
& +\beta_{t} H_{i} \sum_{j=1}^{m}\left(1-u_{i j}\right) I_{j},
\end{aligned}
$$

with nonnegative initial conditions given as $H_{i 0}, I_{i 0}$, and $N=$ $\sum_{i=1}^{m} H_{i}+I_{i}, i, j=1,2, \ldots, m$.

The term $\left(1-u_{1}\right) \theta_{i} I_{i}$ represents the proportion of healthy suckers that are recruited into healthy population arising from infected suckers given application of control. With $u_{1}(t)=1$, only healthy plants are maintained in the plantation. Thus, $u_{1}, u_{2}, u_{3}, u_{i j}=1$ indicated full effort placed in the implementing of controls while $u_{1}, u_{2}, u_{3}, u_{i j}=0$ indicated failure to implement the controls. The objective function to be minimized is given as

$$
\begin{gathered}
J(\bar{u}(\cdot))=\int_{0}^{t_{f}}\left[\sum_{i=1}^{m} A_{i} I_{i}+w_{1} u_{1}(t)+w_{2} u_{2}(t)\right. \\
\left.+\frac{1}{2}\left(w_{3} u_{3}^{2}(t)+\sum_{i=1}^{m} \sum_{j=1}^{m} w_{i j} u_{i j}^{2}(t)\right)\right] d t
\end{gathered}
$$

with $t_{f}$ being the maximum time for which the control practices are implemented.

The constants $A_{i}$ are the weight constants on the infected plants of cultivar $i$ while $w_{1} u_{1}(t), w_{2} u_{2}(t),(1 / 2) w_{3} u_{3}^{2}(t)$, and $(1 / 2) w_{i j} u_{i j}^{2}(t)$ are the corresponding costs for controls $u_{1}, u_{2}, u_{3}$, and $u_{i j}$, respectively, in terms of labor or cash. The costs associated with use of clean planting materials $\left(u_{1}\right)$ and debudding $\left(u_{2}\right)$ are directly proportional to the rates at which these controls are implemented. Thus, it is reasonable to consider the objective function with linear controls $u_{1}, u_{2}$ strategy. Later these will be adjusted to include quadratic control terms to make the problem mathematically more tractable. The quadratic cost functions are used as the simplest form of describing nonlinear costs involved in implementation of the controls disinfection of tools $\left(u_{i j}\right)$ and roguing $u_{3}[10]$.

The target is to determine an optimal control solution $\bar{u}^{*}=\left(u_{1}^{*}, u_{2}^{*}, u_{3}^{*}, u_{i j}^{*}\right)$ for $i, j=1,2, \ldots, m$ with their corresponding state solution which minimizes the objective function subject to the system on the defined control space:

$$
\begin{aligned}
\Omega= & \left\{(\bar{u}) \mid \bar{u}(t) \text { is Lebesgue measurable on }\left[0, t_{f}\right]\right. \\
& \text { with } 0 \leq \bar{u}(t) \leq 1\} .
\end{aligned}
$$

Thus,

$$
\begin{aligned}
J\left(\bar{u}^{*}\right)= & \min _{\bar{u} \in \Omega} J(\bar{u}) \\
& \text { where } \bar{u}=\left(u_{1}, u_{2}, u_{3}, u_{i j}\right), i, j=1,2, \ldots, m .
\end{aligned}
$$


Applying all the control options would be the most rapid method in stopping the spread of BXW but this is often too costly [9]. Therefore, here we determine combinations of control measures that would effectively lead to disease eradication at minimum total cost of implementation.

\section{Optimal Control Model: Special Case of Two Cultivars}

To analyze the optimal control model, we consider a special case whereby, on a particular plantation, only two cultivars, that is, AAA-genome cultivars $(i=1)$ and ABB-genome cultivar $(i=2)$, are considered. This is justified by the common practice in the affected areas of growing these two types, one for food and trade and one for beer.

First, we formulate our optimal control problem considering linear cost function for use of clean planting material and debudding controls with an intention of determining $0 \leq u_{1}^{*} \leq 1,0 \leq u_{2}^{*} \leq 1,0 \leq u_{3}^{*} \leq 1,0 \leq u_{i j}^{*} \leq 1$, for $i, j=1,2$ for $t=\left[0, t_{f}\right]$ that minimize

$$
\begin{aligned}
& J(\bar{u})=\int_{0}^{t_{f}}\left[A_{1} I_{1}+A_{2} I_{2}+w_{1} u_{1}(t)+w_{2} u_{2}(t)\right. \\
& \left.+\frac{1}{2}\left(w_{3} u_{3}^{2}(t)+w_{i j} u_{i j}^{2}(t)\right)\right] d t
\end{aligned}
$$

subject to

$$
\begin{aligned}
\frac{d H_{1}}{d t}= & r\left(1-\frac{N_{1}}{K}\right)\left(H_{1}+\left(1-u_{1}\right) \theta_{1} I_{1}\right) \\
& -\beta_{t} H_{1}\left(\left(1-u_{11}\right) I_{1}+\left(1-u_{12}\right) I_{2}\right) \\
& -\frac{\left(1-u_{2}\right) \beta_{v} H_{1}}{N}\left(I_{1}+I_{2}\right), \\
\frac{d I_{1}}{d t}= & r\left(1-\frac{N_{1}}{K}\right)\left(1-\left(1-u_{1}\right) \theta_{1}\right) I_{1} \\
& +\beta_{t} H_{1}\left(\left(1-u_{11}\right) I_{1}+\left(1-u_{12}\right) I_{2}\right) \\
& +\frac{\left(1-u_{2}\right) \beta_{v} H_{1}}{N}\left(I_{1}+I_{2}\right)-\delta_{1} u_{3} I_{1} \\
\frac{d H_{2}}{d t}= & r\left(1-\frac{N_{2}}{K}\right)\left(H_{2}+\left(1-u_{1}\right) \theta_{2} I_{2}\right) \\
& -\beta_{t} H_{2}\left(\left(1-u_{21}\right) I_{1}+\left(1-u_{22}\right) I_{2}\right) \\
& -\frac{\left(1-u_{2}\right) \beta_{v} H_{2}}{N}\left(I_{1}+I_{2}\right) \\
& +\frac{\left(1-u_{2}\right) \beta_{v} H_{2}}{N}\left(I_{1}+I_{2}\right)-\delta_{2} u_{3} I_{2} . \\
\frac{d I_{2}}{d t}= & r\left(1-\frac{N_{2}}{K}\right)\left(1-\left(1-u_{1}\right) \theta_{2}\right) I_{2} \\
& \left(\left(1-u_{21}\right) I_{1}+\left(1-u_{22}\right) I_{2}\right) \\
& \left(H_{2}\right)
\end{aligned}
$$

From system (6), it is noted that, in the absence of disease, no control practice is implemented $\left(I_{i}=0, u_{1}=0, u_{2}=0\right.$, $\left.u_{3}=0, u_{i j}=0\right)$. The population growth per cultivar is determined by

$$
\frac{d N_{i}}{d t}=r\left(1-\frac{N_{i}}{K}\right) N_{i} \leq 0 \quad \text { if } N_{i}(t) \geq K .
$$

Moreover, by solving for $N_{i}(t)$, we obtain $N_{i}(t)=K N_{i}(0) /$ $\left(K e^{-r t}+N_{i}(0)\left(1-e^{-r t}\right)\right) \rightarrow K$ as $t \rightarrow \infty$.

Thus, given $H_{i}(0) \geq 0, I_{i}(0) \geq 0$, for $i=1,2$, the state solutions $H_{i}(t), I_{i}(t)$ are positively invariant for all $t \geq 0$. That is, the set

$$
\begin{aligned}
X & =\left\{\left(H_{i}, I_{i}\right) \in \mathbf{R}_{+}^{4} \mid H_{i} \geq 0, I_{i} \geq 0, H_{i}+I_{i} \leq K \text { for } i\right. \\
& =1,2\}
\end{aligned}
$$

is positively invariant. Furthermore, the following holds:

$$
\lim _{t \rightarrow \infty} N_{i}(t)=K \quad \text { for } i=1,2
$$

3.1. Existence of the Optimal Control Solution. Consider linear controls $\left(u_{1}\right)$ and $\left(u_{2}\right)$ and quadratic controls $u_{i j}$ and $u_{3}$ such that the costs associated with controls $u_{1}, u_{2}, u_{i j}$, and $u_{3}$ are $w_{1} u_{1}(t), w_{2} u_{2}(t),(1 / 2) w_{3} u_{3}^{2}(t)$, and $(1 / 2) w_{i j} u_{i j}^{2}(t)$, respectively. We show that the optimal control solution exists and then we characterize the system using Pontryagin's Maximum Principle $[17,20]$ to obtain the optimal control solution.

Theorem 1. There exists an optimal control solution

$$
\bar{u}^{*}=\left(u_{1}^{*}, u_{2}^{*}, u_{3}^{*}, u_{11}^{*}, u_{12}^{*}, u_{21}^{*}, u_{22}^{*}\right)
$$

for objective function (5) subject to model system (6).

Proof. Let $x(t)$ denote the state variables and $u(t)$ denote the control variables. Let the integrand for the objective function be defined as

$$
\begin{aligned}
L(t, x(t), u(t))= & A_{1} I_{1}+A_{2} I_{2}+w_{1} u_{1}(t)+w_{2} u_{2}(t) \\
& +\frac{1}{2}\left(w_{3} u_{3}^{2}(t)+w_{i j} u_{i j}^{2}(t)\right) .
\end{aligned}
$$

To prove existence of the optimal control solution, we apply Theorem 4.1 in [21] by checking the following assumptions.

(A1) The set of control and corresponding variables is nonempty.

(A2) The control set $\Omega$ is closed and convex.

(A3) The right-hand side of the state system is bounded above by a sum of bounded control and the state and can be written as a linear function with coefficients dependent on time and state.

(A4) The integrand is a convex function on $\Omega$.

(A5) There exists constant $c_{1}, c_{2}>0$ and $\tau>1$ such that the integrand satisfies

$$
L(t, x(t), u(t)) \geq c_{1}|u|^{\tau / 2}-c_{2} .
$$


Since the equations of system (6) have bounded coefficients and the solution exists in finite time intervals, Theorem 9.2.1 in [22] guarantees the existence of the solution to the model system which gives assumption (A1). By definition of the set $\Omega$, (A2) is also satisfied. From system (6), the righthand side of the equations is continuous and linear in the controls indicating that it can be written as $\bar{f}(t, \bar{x}, \bar{u})=$ $\bar{g}(t, \bar{x})+\bar{h}(t, \bar{x}) \bar{u}$, where $\bar{x}=\left(H_{1}, I_{1}, H_{2}, I_{2}\right)$ and $\bar{u}=$ $\left(u_{1}, u_{2}, u_{3}, u_{11}, u_{12}, u_{21}, u_{22}\right)$. By the boundedness of the solution, $|\bar{f}(t, \bar{x}, \bar{u})| \leq \bar{B}(|\bar{x}|+|\bar{u}|)$ for $0 \leq t \leq t_{f}$. Thus (A3) is also satisfied.

For (A4), the integrand $L(t, x(t), u(t))$ is convex; that is, it can be easily shown given $p \in(0,1)$. Let $u(t)$ and $\nu(t)$ be two vectors in the integrand; then

$$
\begin{aligned}
& L(t, x(t),(1-p) u(t)+p v(t))-(1-p) L(t, x(t), \\
& u(t))-p L(t, x(t), v(t))=\frac{1}{2}\left[\left(p^{2}-p\right)\right. \\
& \cdot\left(w_{3}\left(u_{3}-v_{3}\right)^{2}+w_{11}\left(u_{11}-v_{11}\right)^{2}\right. \\
& +w_{12}\left(u_{12}-v_{12}\right)^{2}+w_{21}\left(u_{21}-v_{21}\right)^{2} \\
& \left.\left.+w_{22}\left(u_{22}-v_{22}\right)^{2}\right)\right]<0 \\
& \quad \text { since } p \in(0,1) \longrightarrow p^{2}<p .
\end{aligned}
$$

Lastly there exist constants $c_{1}, c_{2}>0$ and $\tau>1$ such that

$$
\begin{aligned}
L(t, x(t), u(t)) \geq & c_{1}\left(\left|u_{1}\right|^{2}+\left|u_{2}\right|^{2}+\cdots+\left|u_{22}\right|^{2}\right)^{\tau / 2} \\
& -c_{2}=c_{1}|\bar{u}|^{\tau / 2}-c_{2} .
\end{aligned}
$$

Thus, assumption (A5) is satisfied.

3.1.1. Characterization of the Optimal Control Solution. Pontryagin's Maximum Principle is applied to obtain the necessary conditions that an optimal control pair $\left(x^{*}(t), u^{*}(t)\right)$ must satisfy. The principle converts the optimal control problem given by objective function (5) subject to system (6) into a problem of minimizing the Hamiltonian $H(t, x(t), u(t), \lambda(t))$ with respect to the control set $u(t)$. $\lambda(t)=\left(\lambda_{1}(t), \lambda_{2}(t), \lambda_{3}(t), \lambda_{4}(t)\right)$ is the nonempty adjoint vector function corresponding to the state variables $x(t)=$ $\left(H_{1}(t), I_{1}(t), H_{2}(t), I_{2}(t)\right)$. The necessary conditions to be satisfied are

$$
\begin{aligned}
\frac{d x(t)}{d t} & =\frac{\partial H(t, x(t), u(t), \lambda(t))}{\partial \lambda}, \\
H\left(t, x(t), u^{*}(t), \lambda(t)\right) & \leq H(t, x(t), u(t), \lambda(t)), \\
\forall u \in \Omega & \\
\frac{d \lambda(t)}{d t} & =-\frac{\partial H(t, x(t), u(t), \lambda(t))}{\partial x},
\end{aligned}
$$

with the transversality condition $\lambda\left(t_{f}\right)=0$.
We define the Hamiltonian equation

$$
\begin{aligned}
& H=A_{1} I_{1}+A_{2} I_{2}+w_{1} u_{1}+w_{2} u_{2}+\frac{1}{2}\left(w_{3} u_{3}^{2}+w_{11} u_{11}^{2}\right. \\
& \left.+w_{12} u_{12}^{2}+w_{21} u_{21}^{2}+w_{22} u_{22}^{2}\right) \\
& +\lambda_{1}\left\{\left(1-\frac{N_{1}}{K}\right)\left(H_{1}+\left(1-u_{1}\right) \theta_{1} I_{1}\right)\right. \\
& -\beta_{t} H_{1}\left(\left(1-u_{11}\right) I_{1}+\left(1-u_{12}\right) I_{2}\right) \\
& \left.-\frac{\left(1-u_{2}\right) \beta_{v} H_{1}}{N}\left(I_{1}+I_{2}\right)\right\} \\
& +\lambda_{2}\left\{r\left(1-\frac{N_{1}}{K}\right)\left(1-\left(1-u_{1}\right) \theta_{1}\right) I_{1}-\delta_{1} u_{3} I_{1}\right. \\
& +\frac{\left(1-u_{2}\right) \beta_{v} H_{1}}{N}\left(I_{1}+I_{2}\right) \\
& \left.+\beta_{t} H_{1}\left(\left(1-u_{11}\right) I_{1}+\left(1-u_{12}\right) I_{2}\right)\right\} \\
& +\lambda_{3}\left\{r\left(1-\frac{N_{2}}{K}\right)\left(H_{2}+\left(1-u_{1}\right) \theta_{2} I_{2}\right)\right. \\
& -\beta_{t} H_{2}\left(\left(1-u_{21}\right) I_{1}+\left(1-u_{22}\right) I_{2}\right) \\
& \left.-\frac{\left(1-u_{2}\right) \beta_{v} H_{2}}{N}\left(I_{1}+I_{2}\right)\right\} \\
& +\lambda_{4}\left\{r\left(1-\frac{N_{2}}{K}\right)\left(1-\left(1-u_{1}\right) \theta_{2}\right) I_{2}-\delta_{2} u_{3} I_{2}\right. \\
& +\beta_{t} H_{2}\left(\left(1-u_{21}\right) I_{1}+\left(1-u_{22}\right) I_{2}\right) \\
& \left.+\frac{\left(1-u_{2}\right) \beta_{v} H_{2}}{N}\left(I_{1}+I_{2}\right)\right\} \text {. }
\end{aligned}
$$

The differential equations governing the adjoint variables are obtained by differentiating the Hamiltonian $H$ with respect to the state variables. The adjoint system is obtained by determining

$$
\begin{aligned}
\frac{d \lambda_{1}}{d t} & =\frac{\partial H}{\partial H_{1}} \\
\frac{d \lambda_{2}}{d t} & =\frac{\partial H}{\partial I_{1}} \\
\frac{d \lambda_{3}}{d t} & =\frac{\partial H}{\partial H_{2}} \\
\frac{d \lambda_{4}}{d t} & =\frac{\partial H}{\partial I_{4}}
\end{aligned}
$$

written as

$$
\begin{aligned}
\frac{d \lambda_{1}}{d t}= & \frac{\xi_{2}}{N}\left[H_{2}\left(\lambda_{4}-\lambda_{3}\right)+H_{1}\left(\lambda_{2}-\lambda_{1}\right)\right] \\
& -\left[\xi_{11}+\xi_{2}+\xi_{3}\right]\left(\lambda_{2}-\lambda_{1}\right) \\
& +\frac{r}{K}\left(I_{1} \lambda_{2}+H_{1} \lambda_{1}\right)-r \lambda_{1}\left(1-\frac{N_{1}}{K}\right)
\end{aligned}
$$




$$
\begin{aligned}
& \frac{d \lambda_{2}}{d t}=-A_{1} \\
& +\left[\frac{\xi_{2}}{N}-\xi_{6}\right]\left[H_{2}\left(\lambda_{4}-\lambda_{3}\right)+H_{1}\left(\lambda_{2}-\lambda_{1}\right)\right] \\
& +\delta_{1} u_{3} \lambda_{2}+\frac{r}{K}\left(\lambda_{1} H_{1}+\lambda_{2} I_{1}\right) \\
& -\left(\xi_{3}+C_{11}\right)\left(\lambda_{2}-\lambda_{1}\right)-C_{21}\left(\lambda_{4}-\lambda_{3}\right) \\
& -r\left(1-\frac{N}{K}\right)\left[\lambda_{2}-\left(1-u_{1}\right) \theta_{1}\left(\lambda_{2}-\lambda_{1}\right)\right] \\
& \frac{d \lambda_{3}}{d t}=\frac{\xi_{2}}{N}\left[H_{1}\left(\lambda_{2}-\lambda_{1}\right)+H_{2}\left(\lambda_{4}-\lambda_{3}\right)\right] \\
& -\left(\xi_{12}+\xi_{2}+\xi_{4}\right)\left(\lambda_{4}-\lambda_{3}\right) \\
& +\frac{r}{K}\left(\lambda_{3} H_{2}+\lambda_{4} I_{2}\right)-\lambda_{3} r\left(1-\frac{N_{2}}{K}\right) \\
& \frac{d \lambda_{4}}{d t}=-A_{2} \\
& +\left[\frac{\xi_{2}}{N}-\xi_{6}\right]\left[H_{1}\left(\lambda_{2}-\lambda_{1}\right)+H_{2}\left(\lambda_{4}-\lambda_{3}\right)\right] \\
& +\delta_{2} u_{3} \lambda_{4}+\frac{r}{K}\left(\lambda_{3} H_{2}+\lambda_{4} I_{2}\right) \\
& -C_{12}\left(\lambda_{2}-\lambda_{1}\right)-\left(\xi_{4}+C_{22}\right)\left(\lambda_{4}-\lambda_{3}\right) \\
& -r\left(1-\frac{N}{K}\right)\left[\lambda_{4}-\left(1-u_{1}\right) \theta_{2}\left(\lambda_{4}-\lambda_{3}\right)\right] \text {, }
\end{aligned}
$$

where

$$
\begin{aligned}
\xi_{11} & =\beta_{t}\left(I_{1}\left(1-u_{11}\right)+I_{2}\left(1-u_{12}\right)\right) \\
\xi_{12} & =\beta_{t}\left(I_{1}\left(1-u_{21}\right)+I_{2}\left(1-u_{22}\right)\right) \\
\xi_{2} & =\frac{\beta_{v}\left(1-u_{2}\right)\left(I_{1}+I_{2}\right)}{N} \\
\xi_{3} & =\frac{r I_{1}\left(1-u_{1}\right) \theta_{1}}{K} \\
\xi_{4} & =\frac{r I_{2}\left(1-u_{1}\right) \theta_{2}}{K} \\
\xi_{6} & =\frac{\left(1-u_{2}\right) \beta_{v}}{N} \\
C_{11} & =H_{1}\left(1-u_{11}\right) \\
C_{12} & =H_{1}\left(1-u_{12}\right) \beta_{t}
\end{aligned}
$$

$$
\begin{aligned}
& C_{21}=H_{2}\left(1-u_{21}\right) \beta_{t} \\
& C_{22}=H_{2}\left(1-u_{22}\right) \beta_{t}
\end{aligned}
$$

with the transversality condition $\lambda_{1}\left(t_{f}\right)=\lambda_{2}\left(t_{f}\right)=$ $\lambda_{3}\left(t_{f}\right)=\lambda_{4}\left(t_{f}\right)=0$. For the optimal controls, we solve for $\left(u_{1}^{*}, u_{2}^{*}, u_{3}^{*}, u_{11}^{*}, u_{12}^{*}, u_{21}^{*}, u_{22}^{*}\right)$ by differentiating $H$ with respect to the respective controls, and considering the bounds, we obtain the optimal control solution as follows.

For the linear controls $u_{1}, u_{2}$, bounded such that $0 \leq$ $u(t) \leq 1$, the optimal control solution is given as

$$
\begin{array}{r}
u_{1}^{*}(t)= \begin{cases}0 & \text { if } \frac{\partial H}{\partial u_{1}}>0 \\
u_{1 s}^{*} & \text { if } \frac{\partial H}{\partial u_{1}}=0 \\
1 & \text { if } \frac{\partial H}{\partial u_{1}}<0\end{cases} \\
u_{2}^{*}(t)= \begin{cases}0 & \text { if } \frac{\partial H}{\partial u_{2}}>0 \\
u_{2 s}^{*} & \text { if } \frac{\partial H}{\partial u_{2}}=0 \\
1 & \text { if } \frac{\partial H}{\partial u_{2}}<0 .\end{cases}
\end{array}
$$

Let $\phi_{1}=\partial H / \partial u_{1}$ and $\phi_{2}=\partial H / \partial u_{2}$ referred to as switching functions for the controls $u_{1}$ and $u_{2}$, respectively. In the region where $\phi_{1}$ and $\phi_{2}$ are not equal to zero, we say that the controls $u_{1}$ and $u_{2}$ are bang-bang controls. In this case, applications of these controls are switched between the lower and the upper bound through the period of implementation. To address the case where $\phi_{1}$ and $\phi_{2}$ are zeros for some time interval $\left\{t_{1}, t_{2}\right\}$, we note that all the derivatives with respect to the corresponding controls vanish in this time interval. The functions $\phi_{1}$ and $\phi_{2}$ are differentiated with respect to time and substitution is consequently done until the controls $u_{1}$ and $u_{2}$, respectively, reappear. The optimal controls are then referred to as singular arcs. The singular arcs $u_{1 s}$ and $u_{2 s}$ are then determined by equating the time derivatives to zero and solving. If the $k$ th derivative of the switching functions $\phi_{1}, \phi_{2}$ is nonzero and the Generalized Legendre Clebsch Condition given by

$$
(-1)^{k} \frac{\partial H}{\partial u_{i}}\left[\frac{\partial^{2 k} \phi_{i}}{\partial t^{2 k}}\right] \geq 0 \text { for } k=0,1,2, \ldots
$$

is satisfied, then the singular controls have order $k$ [23]. Thus, for the system (Section 3), we have the optimal control solution given as

$$
u_{1}^{*}(t)= \begin{cases}0 & \text { if } \phi_{1}>0 \\ u_{1 s}^{*} & \text { if } \phi_{1}=0 \\ 1 & \text { if } \phi_{1}<0\end{cases}
$$

where, $\phi_{1}=\frac{K w_{1}+r\left(N_{1}-K\right) \theta_{1} I_{1}\left(\lambda_{1}^{*}-\lambda_{2}^{*}\right)-r I_{2}\left(N_{2}-K\right)\left(\lambda_{3}^{*}-\lambda_{4}^{*}\right) \theta_{2}}{K}$ 


$$
\begin{aligned}
u_{2}^{*}(t)= \begin{cases}0 & \text { if } \phi_{2}>0 \\
u_{2 s}^{*} & \text { if } \phi_{2}=0 \\
1 & \text { if } \phi_{2}<0\end{cases} \\
\quad \text { where, } \phi_{2}=w_{1}+\frac{\left(I_{1}+I_{2}\right)\left(H_{1}\left(\lambda_{1}^{*}-\lambda_{2}^{*}\right)+H_{2}\left(\lambda_{3}^{*}-\lambda_{4}^{*}\right)\right) \beta_{v}}{N} .
\end{aligned}
$$

For the quadratic controls, given the bound $0 \leq u_{3}, u_{i j} \leq 1$, where $i, j=1,2$, the optimal controls are given as

$$
\begin{aligned}
& u_{3}^{*}(t)= \begin{cases}u_{3}^{*}=0 & \text { if } \frac{\partial H}{\partial u_{3}}<0 \\
0 \leq u_{3}^{*} \leq 1 & \text { if } \frac{\partial H}{\partial u_{1}}=0 \\
u_{3}^{*}=1 & \text { if } \frac{\partial H}{\partial u_{1}}>0\end{cases} \\
& u_{1 j}^{*}(t)= \begin{cases}u_{i j}^{*}=0 & \text { if } \frac{\partial H}{\partial u_{i j}}<0 \\
0 \leq u_{i j}^{*} \leq 1 & \text { if } \frac{\partial H}{\partial u_{i j}}=0 \\
u_{i j}^{*}=1 & \text { if } \frac{\partial H}{\partial u_{i j}}>0 .\end{cases}
\end{aligned}
$$

These can be summarized as

$$
\begin{aligned}
& u_{3}^{*}=\min \left\{1, \max \left\{0, \frac{I_{1} \lambda_{2}^{*} \delta_{1}+I_{2} \lambda_{4}^{*} \delta_{2}}{w_{3}}\right\}\right\} \\
& u_{11}^{*}=\min \left\{1, \max \left\{0, \frac{I_{1} H_{1}\left(\lambda_{2}^{*}-\lambda_{1}^{*}\right) \beta_{t}}{w_{11}}\right\}\right\} \\
& u_{12}^{*}=\min \left\{1, \max \left\{0, \frac{I_{2} H_{1}\left(\lambda_{2}^{*}-\lambda_{1}^{*}\right) \beta_{t}}{w_{12}}\right\}\right\} \\
& u_{21}^{*}=\min \left\{1, \max \left\{0, \frac{I_{1} H_{2}\left(\lambda_{4}^{*}-\lambda_{3}^{*}\right) \beta_{t}}{w_{21}}\right\}\right\} \\
& u_{22}^{*}=\min \left\{1, \max \left\{0, \frac{I_{2} H_{2}\left(\lambda_{4}^{*}-\lambda_{3}^{*}\right) \beta_{t}}{w_{22}}\right\}\right\},
\end{aligned}
$$

where $\lambda_{1}^{*}, \lambda_{2}^{*}, \lambda_{3}^{*}, \lambda_{4}^{*}$ are solutions to the adjoint system (18) with the transversality condition. The optimal controls for use of clean planting materials $\left(u_{1}\right)$ and debudding $\left(u_{2}\right)$ tend to suggest existence of singular arcs. In this case, the controls are implemented whenever the resources are available which better suits application than the bang-bang case where they are just switched between the lower and the upper limit. According to Ledzewicz and Schätler [23], singular controls (if they exist) tend to be either the best (minimizing) or the worst (maximizing) strategies and in either case they are essential in determining the structure of optimal controls. However due to the complexity of the optimal control model, this can not be verified. Thus we consider quadratic terms for all the controls in the objective function for the following reasons: (1) Minimizing linear control terms in the objective function is similar to minimizing quadratic terms. (2) With varying seasons, the cost associated with implementation of these control may vary unproportionally to the rate of implementation.

3.1.2. Quadratic Control Terms for Debudding and Use of Clean Planting Materials. Considering quadratic terms for the objective function to be minimized and by applying Pontryagin's Maximum Principle, we have the following Hamiltonian function:

$$
\begin{aligned}
H & =A_{1} I_{1}+A_{2} I_{2}+\frac{1}{2}\left(w_{1} u_{1}^{2}+w_{2} u_{2}^{2}+w_{3} u_{3}^{2}\right. \\
& \left.+w_{11} u_{11}^{2}+w_{12} u_{12}^{2}+w_{21} u_{21}^{2}+w_{22} u_{22}^{2}\right) \\
& +\lambda_{1}\left\{\left(1-\frac{N_{1}}{K}\right)\left(H_{1}+\left(1-u_{1}\right) \theta_{1} I_{1}\right)\right. \\
& -\beta_{t} H_{1}\left(\left(1-u_{11}\right) I_{1}+\left(1-u_{12}\right) I_{2}\right) \\
& \left.-\frac{\left(1-u_{2}\right) \beta_{v} H_{1}}{N}\left(I_{1}+I_{2}\right)\right\} \\
& +\lambda_{2}\left\{r\left(1-\frac{N_{1}}{K}\right)\left(1-\left(1-u_{1}\right) \theta_{1}\right) I_{1}-\delta_{1} u_{3} I_{1}\right. \\
& +\frac{\left(1-u_{2}\right) \beta_{v} H_{1}}{N}\left(I_{1}+I_{2}\right) \\
& +\beta_{t} H_{2}\left(\left(1-u_{21}\right) I_{1}+\left(1-u_{22}\right) I_{2}\right) \\
& \left.+\beta_{t} H_{1}\left(\left(1-u_{11}\right) I_{1}+\left(1-u_{12}\right) I_{2}\right)\right\} \\
& +\beta_{t} H_{2}\left\{r\left(1-\frac{N_{2}}{K}\right)\left(I_{1}+I_{2}\right)\right\} . \\
& +\left(1-u_{2}\right) \beta_{v} H_{2} \\
& \left.\left.+I_{1}\right) I_{1}+\left(1-u_{22}\right) I_{2}\right) \\
& \left.\left.+I_{2} I_{2}\right)\right\}
\end{aligned}
$$

The existence of controls in this case can be proved using similar techniques as in Theorem 1 . The adjoint variables are 
the same as system (18). Thus the optimal control solution can then be given as

$$
\begin{aligned}
& u_{1}^{*}=\min \left\{1, \max \left\{0, \frac{r\left(K-N_{1}\right) \theta_{1} I_{1}\left(\lambda_{1}^{*}-\lambda_{2}^{*}\right)-r I_{2}\left(K-N_{2}\right)\left(\lambda_{3}^{*}-\lambda_{4}^{*}\right) \theta_{2}}{K w_{1}}\right\}\right\} \\
& u_{2}^{*}=\min \left\{1, \max \left\{0,-\frac{\left(I_{1}+I_{2}\right)\left(H_{1}\left(\lambda_{1}^{*}-\lambda_{2}^{*}\right)+H_{2}\left(\lambda_{3}^{*}-\lambda_{4}^{*}\right)\right) \beta_{v}}{N w_{2}}\right\}\right\} \\
& u_{3}^{*}=\min \left\{1, \max \left\{0, \frac{I_{1} \lambda_{2}^{*} \delta_{1}+I_{2} \lambda_{4}^{*} \delta_{2}}{w_{3}}\right\}\right\} \\
& u_{11}^{*}=\min \left\{1, \max \left\{0, \frac{I_{1} H_{1}\left(\lambda_{2}^{*}-\lambda_{1}^{*}\right) \beta_{t}}{w_{11}}\right\}\right\} \\
& u_{12}^{*}=\min \left\{1, \max \left\{0, \frac{I_{2} H_{1}\left(\lambda_{2}^{*}-\lambda_{1}^{*}\right) \beta_{t}}{w_{12}}\right\}\right\} \\
& u_{21}^{*}=\min \left\{1, \max \left\{0, \frac{I_{1} H_{2}\left(\lambda_{4}^{*}-\lambda_{3}^{*}\right) \beta_{t}}{w_{21}}\right\}\right\} \\
& u_{22}^{*}=\min \left\{1, \max \left\{0, \frac{I_{2} H_{2}\left(\lambda_{4}^{*}-\lambda_{3}^{*}\right) \beta_{t}}{w_{22}}\right\}\right\} .
\end{aligned}
$$

The optimal control characterizations are inversely proportional to the associated weighted costs of implementation indicating that the effectiveness of the control strategy greatly depends on the necessary costs.

Next we solve numerically the optimality system consisting of state system (6) with corresponding initial conditions, adjoint system (18) with the transversality condition, and optimal control characterization assuming the costs terms associated with the controls are quadratic (26).

3.2. Numerical Simulation. In this section, numerical results for the optimal control model given in Section 3 are studied. The backward-forward sweep method with the fourth-order Runge-Kutta algorithm is applied to solve the optimality system. The algorithm has been implemented by various authors to obtain optimal control solutions of related systems $[11,14,17]$. For easy reference we summarize it below.

(i) Make an initial guess of the control; $\bar{u}=0$ is always sufficient.

(ii) Using the initial conditions $H_{10}, I_{10}, H_{20}, I_{20}$, and the values of $\bar{u}$ solve for $\bar{x}$ forward in time according to the differential equations in the optimality system given.

(iii) Using the transversality condition $\bar{\lambda}\left(t_{f}\right)=0$ and the values of $\bar{x}$ and $\bar{u}$ solve the adjoint system $\bar{\lambda}$ backward in time according to the corresponding differential equations in the optimality system.

(iv) Update the control set $\bar{u}$ by entering the new values of $\bar{x}$ and $\bar{\lambda}$ into the characterization of the optimal control. (v) Verify for convergence the following: if the values obtained are sufficiently close to the corresponding ones in the previous iterations, then the output of the current values is the optimal control solution; otherwise return to step (ii).

This algorithm was implemented in Matlab by modifying the optimal control code presented by [17].

The numerical values used for the numerical simulation as reflected in Table 1 are obtained from literature and some are selected from given ranges based on the banana growing system in Uganda, for which the feasible solution is guaranteed.

According to the guide by [24], the maintenance rate, $r$, is considered to range between 3 between 5 stems of varying ages per mat. It is noted that the smaller the number of stems the bigger the bunch. Also, for a new plantation, the carrying capacity $K$ of stems per hectare given $3 \mathrm{~m} \times 3 \mathrm{~m}$ spacing is assumed to be 750-900 mats [24]. This means, for a wellestablished plantation, the average carrying capacity would range from 2250 to 4500 stems. Roguing by removal of only the infected stem at stool level or by removal of the entire mat depending on the severity of the disease is considered to range between 0 and 1 per month per hectare per infected stem. The proportion of healthy suckers arising from infected mats is also assumed to range between 0 and 1 . Transmission by tools is dependent on the activities being carried out within the plantation. For instance, harvesting of the leaf might require a single cut as compared to desuckering which requires several cuts and exposes the attached mat to infection. Accordingly, we assume the rate of tool-mediated transmission to range between 0 and 0.002 . The given range guarantees existence 
TABLE 1: Values used for model simulations.

\begin{tabular}{|c|c|c|c|c|}
\hline Parameter & Range & Value & Unit & Source \\
\hline$r$ & $3-5$ & 3 & Month $^{-1}$ & Assumed \\
\hline$\delta_{1}$ & $0-1$ & 0.1 & Month $^{-1}$ & Assumed \\
\hline$\delta_{2}$ & $0-1$ & 0.1 & Month $^{-1}$ & Assumed \\
\hline$\theta_{1}$ & $0-1$ & 0.9997 & Month $^{-1}$ & [18] \\
\hline$\theta_{2}$ & $0-1$ & 0.9998 & Month $^{-1}$ & [18] \\
\hline$\beta_{v}$ & & 2.031 & Month $^{-1}$ & {$[18]$} \\
\hline$\beta_{t}$ & $0-0.02$ & 0.00002 & Month $^{-1}$ & Assumed \\
\hline K & $1000-4500$ & 1000 & $\mathrm{ha}^{-1}$ & Assumed \\
\hline
\end{tabular}

TABLE 2: Table showing costs of implementations of control options [19].

\begin{tabular}{lcc}
\hline Control practice & Control option & Cost per hectare (Ug.Sh) \\
\hline Use of clean planting materials & $u_{1}$ & $1,454,500$ \\
Disinfection of tools & $u_{i j}, i, j=1,2$ & 12,000 \\
Debudding & $u_{2}$ & 72,000 \\
Roguing & $u_{3}$ & $1,615,500$ \\
\hline
\end{tabular}

of the optimal solution if other factors are kept constant. The rest of the parameters are as given in [18].

The choice of the weight constants is intended to reflect the cash and labor involved in the control measure. According to the study by Sebikari [19], use of clean planting materials and roguing was more expensive than debudding and disinfection of tools. In Table 2, the costs for implementation of these controls are given.

The low cost for disinfection of tools was due to the fact that most farmers use fire frames made from collected firewood instead of JIK. Additionally, the required labor for activities like disinfection of tools and debudding is mainly from family members which makes these activities less expensive. Thus, we consider reasonable ratios as weights such that $A_{1}=50, A_{2}=50, w_{1}=1212, w_{2}=6, w 3=1346$, and $w_{11}=w 12=w_{21}=w_{22}=1$. Assume the control options are implemented for a period of two years such that $t_{f}=24$ (months).

To illustrate the dynamics of disease with and without controls, we consider the following initial conditions: $H_{10}=$ $300, I_{10}=50, H_{20}=300, I_{20}=50$ chosen arbitrary given that the model exhibits global stability behavior (not presented here). The controls are then introduced after one month. We investigate the following combinations of cultural control practices. Note that several combinations of control strategies have been considered. However, only those giving substantial reductions when applied as compared to no control are presented.

\section{(A) Optimal debudding \\ (B) Combination of two controls}

(B1) Optimal debudding and disinfection of tools between cultivars

(B2) Optimal debudding and disinfection of tools within cultivars

(B3) Optimal debudding and roguing

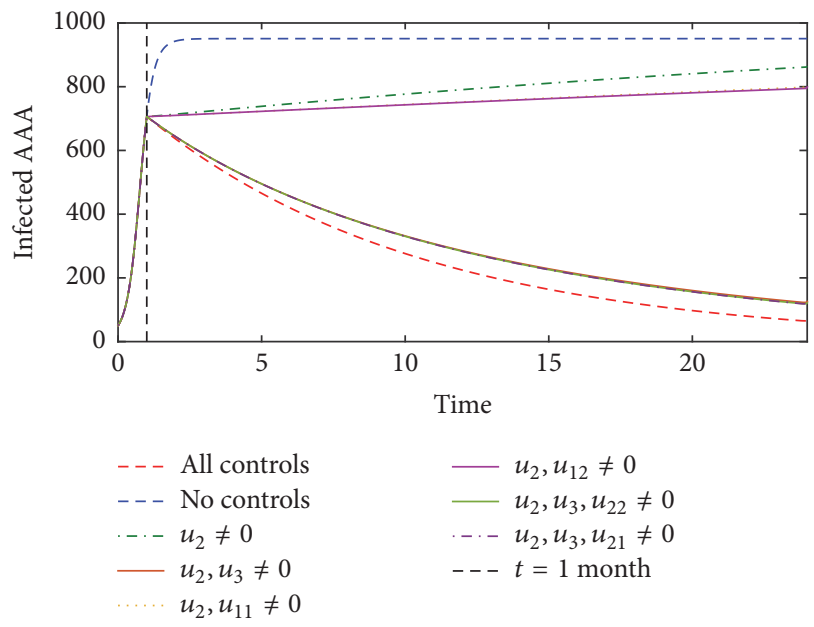

FIGURE 1: Simulation for the number of infected stems for AAAgenome comparing different control strategies after time $t=1$ month applied for a period of 24 months; (A) $\left(u_{2} \neq 0\right),(\mathrm{B} 1)\left(u_{2}, u_{12} \neq\right.$ $0),(\mathrm{B} 2)\left(u_{2}, u_{11} \neq 0\right)$, (B3) $\left(u_{2}, u_{3} \neq 0\right),(\mathrm{C} 1)\left(u_{2}, u_{3}, u_{21} \neq 0\right),(\mathrm{C} 2)$ $\left(u_{2}, u_{3}, u_{22} \neq 0\right)$, and (D) (all controls).

(C) Combination of three control options

(C1) Optimal debudding, disinfection of tools between cultivars, and roguing

(C2) Optimal debudding, disinfection of tools within cultivars, and roguing

(D) Combination of all control options.

In Figures 1 and 2, we present how the incidence of BXW varies from the same starting position with each control strategy. The objective function was optimized using only controls in the given strategy while setting others to zero. 


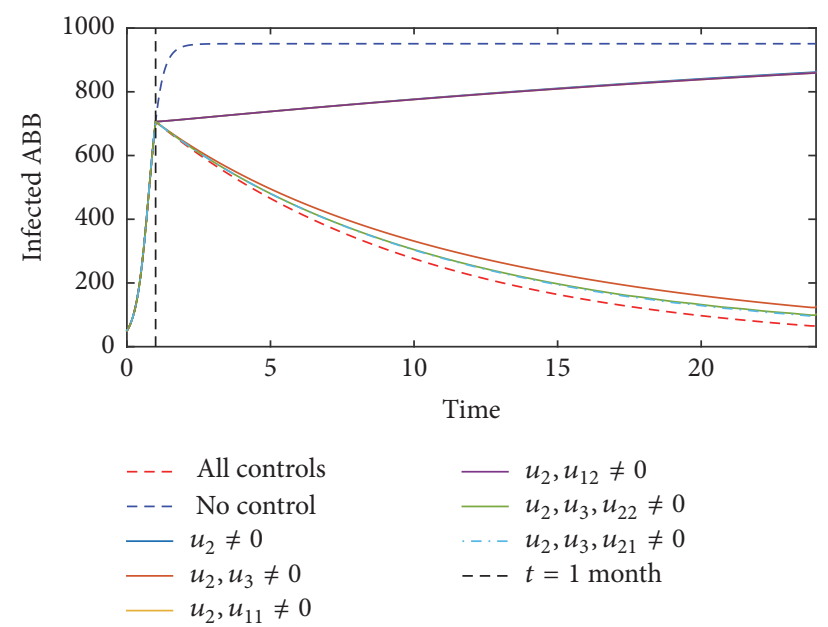

FIgURE 2: Simulation for the number of infected stems for ABBgenome comparing different control strategies after time $t=1$ month applied for a period of 24 months: (A) $\left(u_{2} \neq 0\right),(\mathrm{B} 1)\left(u_{2}, u_{12} \neq\right.$ $0),(\mathrm{B} 2)\left(u_{2}, u_{11} \neq 0\right),(\mathrm{B} 3)\left(u_{2}, u_{3} \neq 0\right),(\mathrm{C} 1)\left(u_{2}, u_{3}, u_{21} \neq 0\right),(\mathrm{C} 2)$ $\left(u_{2}, u_{3}, u_{22} \neq 0\right)$, and (D) (all controls). Parameters are as given in Table 1 .

Strategies (A) (debudding only), (B1) (debudding and disinfection of tools between cultivars), and (B2) (debudding and disinfection of tools within cultivars) are all preventative control practices. In both Figures 1 and 2, the population levels of AAA and ABB infected stems obtained using these control are lower than when no control is applied. Furthermore, we observe that implementation of these controls only reduces the risk of further infection but does not lead to reduction in already infected stems. For example, the number of infected AAA and ABB cultivars was lower by $10 \%$ of the expected number in the absence of control after 20 months of application. It is also noted that disinfection of tools $\left(u_{11}, u_{12}, u_{21}, u_{22}\right)$ led to relatively better results in the AAAgenome cultivar as compared to ABB-genome. This indicates that, in the absence of containment controls, disinfection of tools within AAA-genome cultivars is crucial. From these results, we can conclude that application of only preventative controls delays further infection but, with these parameters, if no other measures are implemented, then uncontrolled endemic levels may be attained asymptotically.

When either of the strategies (B3) (debudding and roguing), (C1) (debudding, roguing, and disinfection of tools between cultivars), and (C2) (a combination of debudding $\left(u_{2}\right)$, roguing $\left(u_{3}\right)$, and disinfection of tools within cultivars) is implemented independently, the number of infected AAA and $\mathrm{ABB}$ stems reduced greatly as compared to the counterparts where only prevention controls are applied. In this case, both prevention and containment controls are applied and as expected the number of infected stems declines by more than $70 \%$ asymptotically to zero. Furthermore, we notice the importance of disinfection of tools among the ABB-genome cultivars. The findings here indicate that disinfection of tools within the highly susceptible cultivars (ABB) and between cultivars is important and reduces the risk of further infection that could arise during roguing and pruning exercises.
Lastly, when all controls are implemented, we observe that the number of infected stems in both cultivars declined rapidly (Figures 1 and 2). That is, AAA and ABB infected stems declined by $90 \%$ of the expected number of stems considered in the absence of the controls.

In Figure 3, we present the control profiles for each strategy defined above. In all cases, we observe that the control profiles for debudding $\left(u_{2}\right)$ and roguing $\left(u_{3}\right)$ are maintained at maximum intensity throughout the period of implementation. The control of use of clean planting materials $\left(u_{1}\right)$ is maintained at the lower limit indicating that this control is neutral and should not be implemented alone but in the combination with other controls. As noted in Figures 1 and 2, application of all controls gave better results as compared to the strategies when $u_{1}$ was set to zero. The control profile for disinfection of tools between and within cultivars $\left(u_{12}\right)$ and $\left(u_{22}\right)$ in strategies (B1) and (B2) is maintained at the upper bound throughout the period of implementation while those in strategies (C1) and (C2) decline and oscillate between the upper and the lower bound after a period of 15 months. This indicates that, in the absence of roguing and considering debudding and disinfection of tools as the only controls applied, maximum effort should be used to reduce new infection. On the contrary, with roguing disinfection of tools can be at maximum intensity when incidence is still high but relaxed and applied at intervals as the infection levels decline.

In Figure 4, numerical results compare control strategies applied at different infection levels $(t=0, t=1$ month, $t=2$ months) with the case of no control. We notice that if control measures are applied at $t=0$ when the prevalence is about $10 \%$, then it would require about 20 months to bring infection below $1 \%$ and 30 months for less than $0.1 \%$. On the contrary, if controls are introduced when the prevalence is about $80 \%(t=1)$, then much more effort and time will be required to attain a prevalence of less than $1 \%$. Also if controls were introduced when the disease was already endemic, then controlling the diseases would become much harder as it would require more time to reduce infection to undetectable levels. Clearly, we notice that disease management and containment are much dependent on the infection level. This implies that once the disease is endemic it may be more cost-effective in clearing the plantation and replanting than in continuously applying the controls which might take up to 5 years to bring disease to undetectable levels.

Additionally, we observe that even when infection is reduced to undetectable levels, that is, $<1 \%$, irrespective of the time of withdrawal of controls, the prevalence increases rapidly towards the endemic equilibrium. In Figure 5, a comparison of the cases where controls are completely withdrawn and when few are maintained is presented after infection is $<1 \%$. We observe that the number of infected stems continues to decline for the case where controls are just reduced compared with the case when they are completely withdrawn. This implies continuous monitoring and application of controls when necessary should be emphasized even when infection levels are undetectable to eliminate cases of resurgence within or outside plantations. 

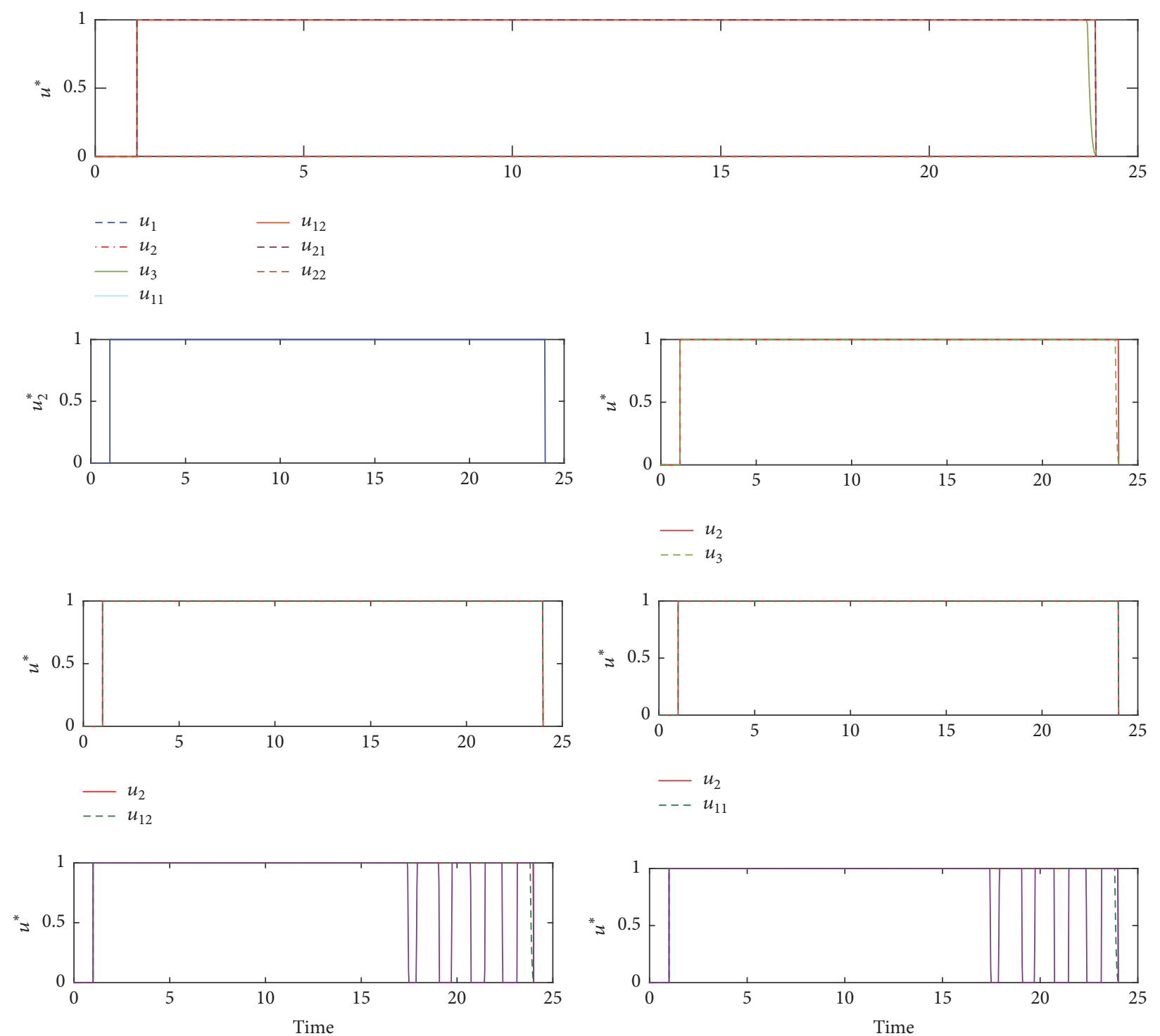

$$
\begin{array}{ll}
- & u_{2} \\
--- & u_{3} \\
- & u_{22}
\end{array}
$$

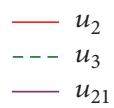

Figure 3: Plots showing control profiles for the different strategies (D), (A), (B1), (B2), (B3), (C1), and (C2), respectively, when quadratic cost terms are considered. The graphs are maintained at maximum value in all strategies for debudding and roguing. Parameters are as given in Table 1.

\section{Conclusions}

In this paper, a deterministic model for optimal control of BXW within a mixed cultivar plantation is derived and analyzed. The possible modes of spread are incorporated including inflorescence infection and use of infected planting materials obtained via mother-to-sucker and tool-mediated spread were considered. The model focuses on the interplay of control options such as debudding, use of clean planting material, disinfection of tools, and roguing as control measures of BXW.

At first, linear cost terms for the debudding and use of clean planting material controls were considered. This is because the cost of implementation of these controls is directly proportional to the rate of implementation. For the other controls, quadratic cost terms were considered due to the nonlinear changes in their implementations. By applying Pontryagin's Maximum Principle, the necessary conditions for optimal control of BXW were obtained. These conditions suggest possibility of having debudding and use of clean planting materials controls as bang-bang controls within the upper and the lower bounds. They appear also to have singular arcs on a given interval for which controls $u_{1}$ and $u_{2}$ are reactivated whenever necessary although this could not be explicitly verified due to the complexity of the model. 


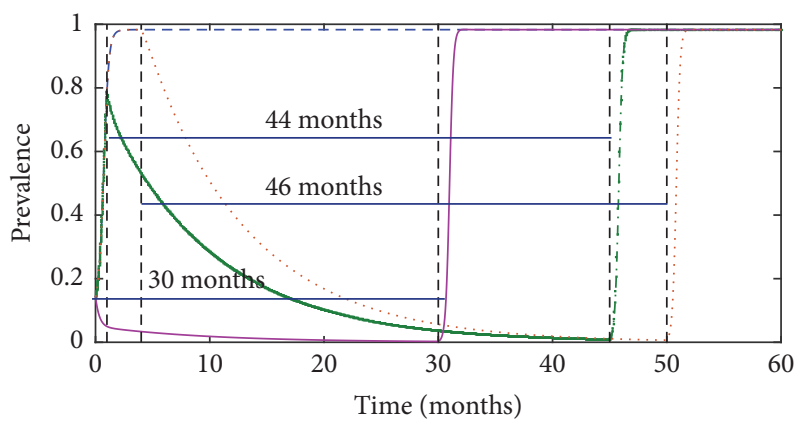

Figure 4: Plot for disease prevalence when all controls are introduced at $t=0,1,2$ months since infection.
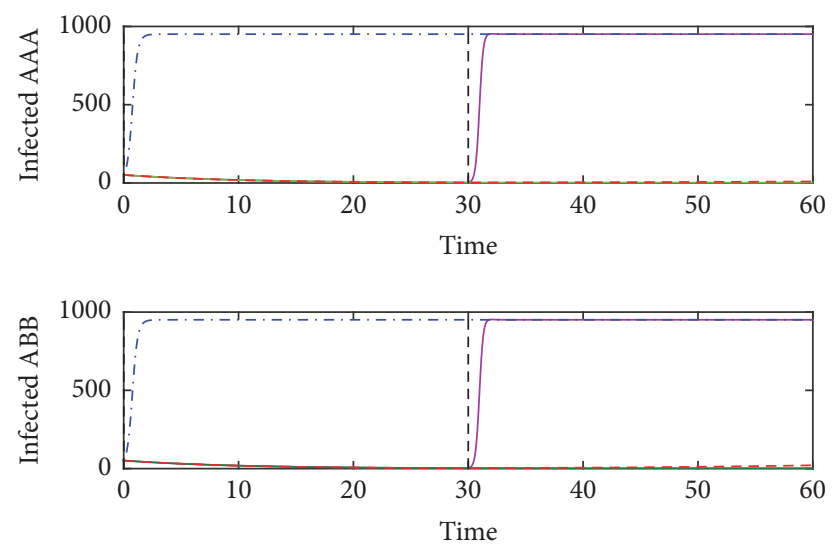

FIGURE 5: Simulation for the number of infected stems for AAAgenome and ABB-genome. The purple line shows, after $t=30$, all controls are withdrawn. Red dashed and green lines indicate the case when only (B1) or (B3) is continued after $t=30$.

To obtain insights into the combination of control options, the linear cost terms for debudding and use of clean planting materials in the objective function were adjusted to include quadratic terms. Using Pontryagin's Maximum Principle and through numerical simulations, it was observed that several combinations of different controls resulted in large reductions in the number of infected BXW stems. From the comparison between the suggested control strategies (see Figures 1 and 2), application of all the controls gave the most significant reduction in the number of infected AAA-genome and $\mathrm{ABB}$-genome cultivars. Our finding further indicates the relevance of applying control measures as soon as infection in observed. We noted that the earlier the control measures are applied, the easier it becomes to manage the disease. Also if disease has reached endemic level, then it is cheaper to clear the plantation than to apply continued controls, since these will take much time and in the long run become costly. Importantly, it was noted that once the controls are withdrawn even with less than $1 \%$ infection level, the disease rapidly increases to endemic level. This calls for emphasis in monitoring and application of controls whenever necessary, even when disease is seemingly eradicated.

Lastly, it is important to note that results obtained are subject to the weight constants chosen and some parameters that vary with season. The true weight constants and parameters would require extensive field work and data collection. It is also important to acknowledge from the model formulation that other factors may influence implementation of the controls including incubation period and biotic and abiotic conditions. However, the results given in this study give insights into how best BXW can be managed.

\section{Conflicts of Interest}

The authors declare that there are no conflicts of interest regarding the publication of this paper.

\section{Acknowledgments}

This research has been funded by Mcknight Foundation and SIDA with additional support from the Roots, Tubers and Banana (RTB) program and Bioversity International. The authors would like to acknowledge the support from SIDA Phase IV Bilateral Programme project 316 "Capacity Building in Mathematics and Its Application."

\section{References}

[1] M. Mwangi and V. Nakato, "Key factors responsible for the xanthomonas wilt epidemic on banana in East and Central Africa," Acta Horticulturae, vol. 828, pp. 395-404, 2009.

[2] E. Karamura, F. Turyagyenda, W. Tinzaara et al., "Xanthomonas Wilt of Bananas in East and Central Africa: Diagnostic and Management Guide," Tech. Rep., Bioversity International, Rome, Italy, 2008.

[3] D. Yirgou and J. E. Bradbury, "A Note on Wilt of Banana Caused by the Enset Wilt Organizm (Xanthomonas musacearum," East African Agricultural and Forestry Journal, pp. 111-114, 1974.

[4] J. Kubiriba and W. Tushemereirwe, "Approaches for the Control of Banana Xanthomonas Wilt in East and Central Africa," African Journal of Plant Science, vol. 8, no. 8, pp. 398-404, 2014.

[5] W. Tushemereirwe, A. Kangire, J. Smith et al., "An Outbreak of Bacterial Wilt on Banana in Uganda," InfoMusa, vol. 12, p. 6, 2003.

[6] W. Tinzaara, C. S. Gold, F. Ssekiwoko, R. Bandyopadhyay, A. Abera, and S. J. Eden-Green, "Role of insects in the transmission of banana bacterial wilt," African Crop Science Journal, vol. 14, no. 2, pp. 105-111, 2006.

[7] F. Bagamba, E. Kikulwe, W. K. Tushemereirwe et al., "Awareness of banana bacterial wilt control in Uganda: 1. Farmers' perspective," African Crop Science Journal, vol. 14, no. 2, pp. 157-164, 2006.

[8] J. Kubiriba, J. Muthomi, V. Ndungo et al., "Strategies for Rehabilitation of Banana Fields Infested with Xanthomonas Campestris pv. musascearum," Journal of Crop Protection, vol. 3, pp. 21-29, 2014.

[9] W. Jogo, E. Karamura, W. Tinzaara, J. Kubiriba, and A. Rietveld, "Determinants of Farm-Level Adoption of Cultural Practices for Banana Xanthomonas Wilt Control in Uganda," Journal of Agricultural Science, vol. 5, no. 7, pp. 70-80, 2013.

[10] E. A. Bakare, A. Nwagwo, and E. Danso-Addo, "Optimal control analysis of an SIR epidemic model with constant recruitment," International Journal of Applied Mathematical Research, vol. 3, no. 3, pp. 273-285, 2014. 
[11] L. Pang, S. Ruan, S. Liu, Z. Zhao, and X. Zhang, "Transmission dynamics and optimal control of measles epidemics," Applied Mathematics and Computation, vol. 256, pp. 131-147, 2015.

[12] G. Devipriya and K. Kalaivani, "Optimal control of multiple transmission of water-borne diseases," International Journal of Mathematics and Mathematical Sciences, vol. 2012, Article ID 421419, pp. 1-16, 2012.

[13] B. Nannyonga, L. S. Luboobi, P. Tushemerirwe, and M. Jablońska-Sabuka, "Using contaminated tools fuels outbreaks of Banana Xanthomonas wilt: An optimal control study within plantations using Runge-Kutta fourth-order algorithms," International Journal of Biomathematics, vol. 8, no. 5, Article ID 1550065, 2015.

[14] H. Namawejje, L. Luboobi, D. Kuznestsov, and E. Wobudeya, "Modeling Optimal Control of Rotavirus Disease with Differrent Control Strategies," Journal of Mathematical and Computational Sciences, vol. 4, pp. 892-914, 2014.

[15] L. C. Evans, An Introduction to Mathematical Optimal Control Theory, University of Califonia, Berkeley, Calif, USA, 2000.

[16] L. Pontryagin, V. G. Boltyanskii, and E. F. Mishchenko, The Mathematical Theory of Optimal Control, Interscience Publishers, Geneva, Switzerland, 1962.

[17] S. M. Lenhart and J. T. Workman, Optimal Control Applied to Biological Models, Taylor \& Francis, Boca Raton, Fla, USA, 2007.

[18] J. Nakakawa, J. Y. Mugisha, M. . Shaw, and E. Karamura, "A mathematical model for the dynamics of banana Xanthomonas wilt with vertical transmission and inflorescence infection," Journal of Biological Systems, vol. 24, no. 1, pp. 147-165, 2016.

[19] M. Sebikari, Aanalysis of Costs Associated with Control of Banana Xanthomonas in Mukono and Luwero Districts Uganda [M.S. thesis], Makerere University, Kampala, Uganda, 2010.

[20] A. B. Kurzhanski, "Pontryagins Maximum Principle," Control Systems, Robotics and Automation, vol. III, Encyclopedia of Life Support Systems.

[21] W. H. Fleming and R. W. Rishel, Deterministic and Stochastic Optimal Control, vol. 1, Springer, New York, NY, USA, 1975.

[22] D. L. Lukes, Differential Equations: Classical to Controlled, Mathematics in Science and Engineering, Academic Press, New York, NY, USA, 1982.

[23] U. Ledzewicz and H. Schätler, "On optimal singular controls for a general SIR-model with vaccination and treatment," Discrete and Continuous Dynamical Systems - Series A, pp. 981-990, 2011.

[24] Banana production guide, http://www.naads.or.ug/files/downloads/Banana. 


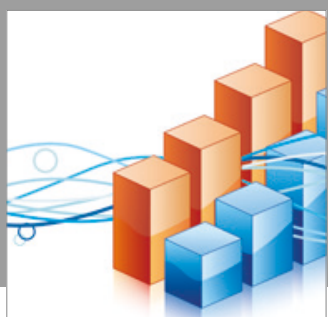

Advances in

Operations Research

vatersals

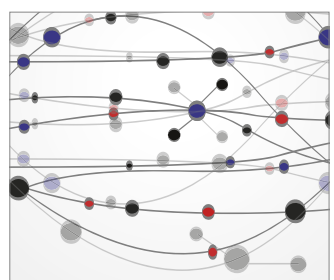

\section{The Scientific} World Journal
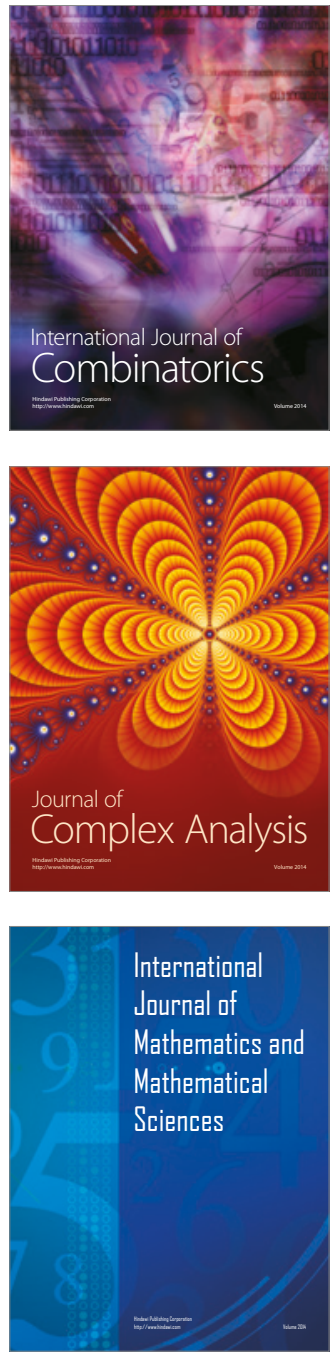
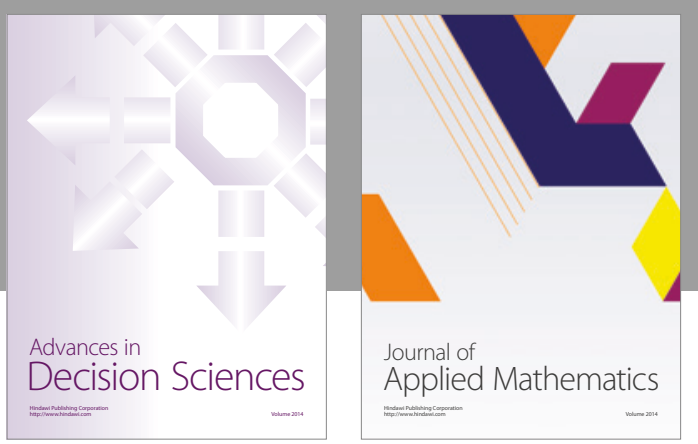

Algebra

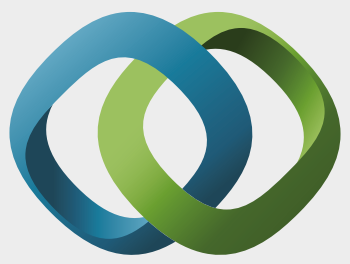

\section{Hindawi}

Submit your manuscripts at

https://www.hindawi.com
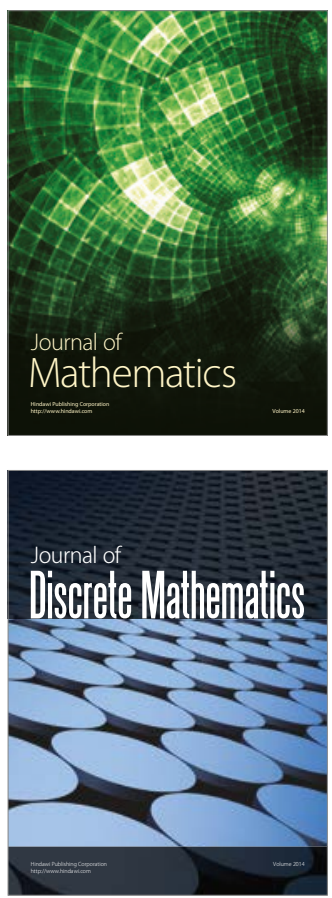

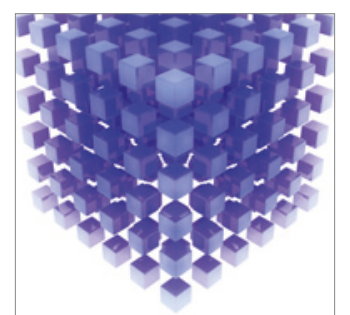

Mathematical Problems in Engineering
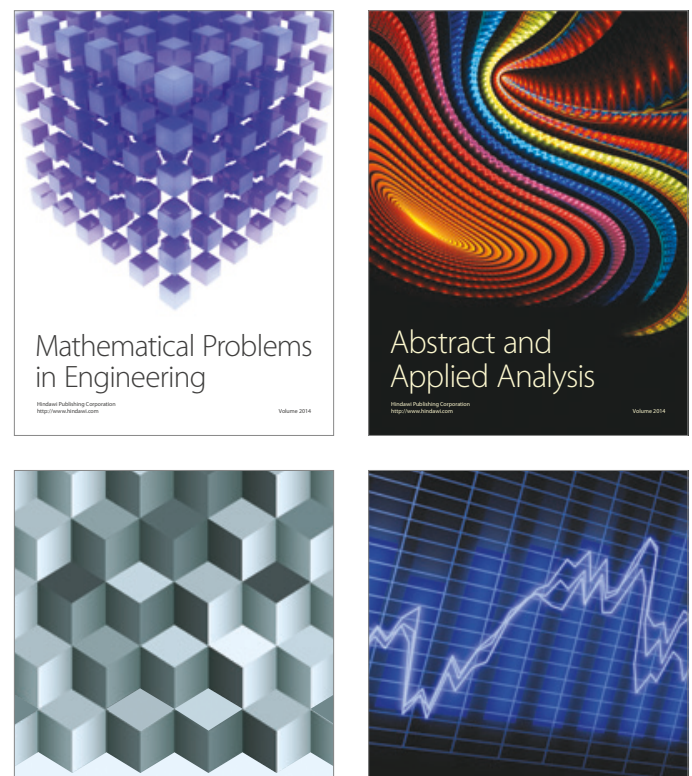

Journal of

Function Spaces

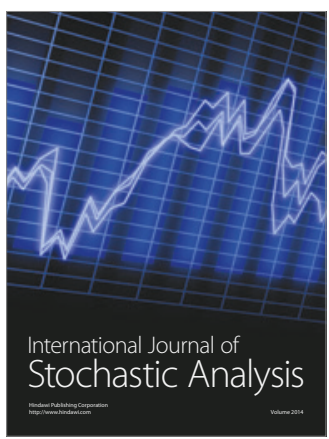

Probability and Statistics
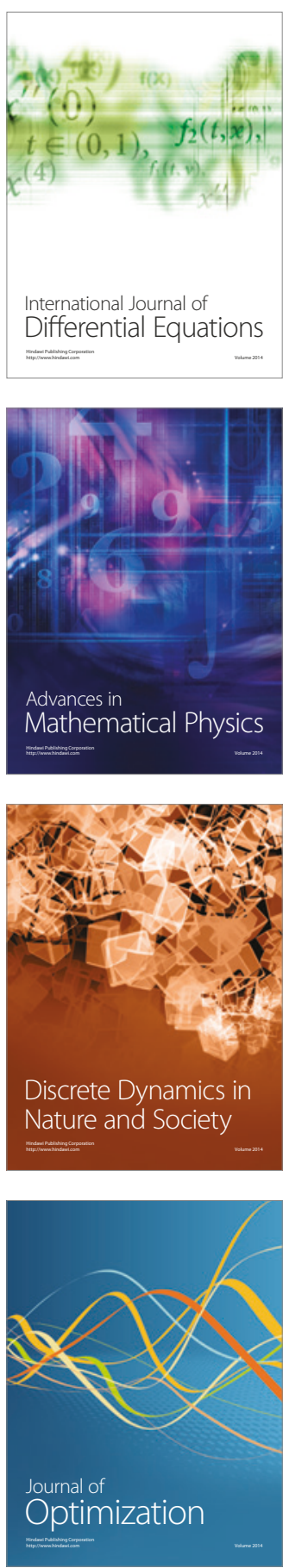